\title{
VLA polarimetry of compact steep spectrum sources
}

\author{
F. Mantovani ${ }^{1}$, W. Junor ${ }^{2}$, R. Fanti ${ }^{1,3}$, L. Padrielli ${ }^{1}$, and D.J. Saikia ${ }^{4}$ \\ 1 Istituto di Radioastronomia del CNR, Bologna, Italy \\ 2 Institute for Astrophysics, University of New Mexico, Albuquerque NM, U.S.A. \\ 3 Dipartimento di Fisica, Universitá degli Studi, Bologna, Italy \\ 4 Tata Institute of Fundamental Research, National Centre for Radio Astrophysics, Pune 411 007, India
}

Received December 24, 1996; accepted February 12, 1997

\begin{abstract}
We present the polarization properties at sub-arcsecond resolutions of 8 Compact Steep-spectrum Sources (CSSs) observed with the VLA A-array at 5, 8.4 and $15 \mathrm{GHz}$. We find that the three most compact sources, two of which are unresolved and have an angular diameter $<100$ milliarcsec are not polarized. Of the remaining 5 , the two associated with quasars have prominent radio jets while the two galaxies and one unidentified object are dominated by their lobe emission. The components of these medium sized objects with median values of percentage polarization of about $9 \%$ at $5 \mathrm{GHz}$ and $11 \%$ at 8.4 GHz, are more strongly polarized than the compact sources and do not show evidence of very large rotation measures.
\end{abstract}

Key words: galaxies: active — galaxies, quasars: radio polarization — interferometry

\section{Introduction}

The Compact Steep-spectrum Sources or CSSs are physically-small objects of sub-galactic dimensions. A large fraction of these, $\sim 70 \%$, which have somewhat symmetric structure and have been named Compact Symmetric Objects, are believed to be the young precursors of the larger sized radio sources (Fanti et al. 1995; Readhead et al. 1996). A minority of CSSs shows a complex morphology or strongly asymmetric emission with respect to the core. The structures and sizes of these are probably affected by the ambient gas near the nucleus of the parent optical object (Pearson et al. 1985; Fanti et al. 1990; Saikia et al. 1995).

Most CSSs show low percentage polarizations (less than $\sim 1 \%$ ) at or below $5 \mathrm{GHz}$ (Saikia et al. 1987). The polarization properties of CSSs at frequencies above 5

Send offprint requests to: F. Mantovani
$\mathrm{GHz}$ have been investigated recently by a number of authors (for example, see Junor et al. 1996 and references therein). Although several well-known members of this class have low rotation measure (RM), radio polarimetric surveys (Kato et al. 1987; Taylor et al. 1992; Inoue et al. 1995) have shown that many of the sources with high $R M\left(>700 \mathrm{rad} \mathrm{m}^{-2}\right)$ are CSSs. VLA observations by Mantovani et al. (1994) and Junor et al. (1996) on selected samples of CSSs have revealed several objects with large $R M$.

In this paper we present VLA A-array observations at 8.4 and $15 \mathrm{GHz}$ of $8 \mathrm{CSSs}, 3$ of which are of subarcsec dimensions while the remaining 5 are medium sized objects. We derive their polarization properties, and compare these with our earlier observations of CSSs. These data complete the information about a sample of sources selected because there were indications (detection of low frequency variability, spectral index turnovers around $100 \mathrm{MHz}$ ) they were compact objects (Mantovani et al. 1992).

\section{VLA observations}

The VLA (Thompson et al. 1980) A-array observations were made in the $X(8.4 \mathrm{GHz})$ and $U(15 \mathrm{GHz})$ bands on 1990 September 10. The data were calibrated in the standard way using VLA calibrators and AIPS procedures. More detailed information on the data analysis can be found in Mantovani et al. (1994). Observations of the same sample of sources were done earlier at $5 \mathrm{GHz}$ with the VLA A-array in 1986 May 30 (Mantovani et al. 1992). However, the polarization data were not presented earlier and are listed here along with the values at the higher frequencies.

\section{VLA structure and polarimetry of CSSs}

\subsection{Derived values}

The values and some of the properties of these sources estimated from the VLA A-configuration images at 5, 8.4 and $15 \mathrm{GHz}$ are presented in Table 1. The contents of Table 1 are as follows: Column 1: source name; Column 2: 
Table 1. Observational parameters and observed properties

\begin{tabular}{|c|c|c|c|c|c|c|c|c|c|c|c|}
\hline Source & $\begin{array}{r}\text { Obs. } \\
\nu \\
\mathrm{MHz} \\
\end{array}$ & $\operatorname{maj}_{/ \prime}$ & $\begin{array}{l}\text { Beam } \\
\text { min. }\end{array}$ & $\begin{array}{r}\mathrm{PA} \\
\circ\end{array}$ & $\begin{array}{c}\sigma_{\mathrm{t}} \\
\mathrm{mJy} / \mathrm{b} \\
\end{array}$ & $\begin{array}{c}\sigma_{\mathrm{p}} \\
\mathrm{mJy} / \mathrm{b}\end{array}$ & $\mathrm{C}$ & $\begin{array}{r}\text { R.A. (B1950) } \\
\text { h m s } \\
\end{array}$ & $\begin{array}{r}\text { Dec.(B1950) } \\
\circ, \prime \prime\end{array}$ & $\begin{array}{r}\text { Flux } \\
\text { peak } \\
\text { mJy/b }\end{array}$ & $\begin{array}{r}\text { Dens. } \\
\text { total } \\
\text { mJy } \\
\end{array}$ \\
\hline \multirow[t]{3}{*}{$\overline{0320+053}$} & $\bar{~} 4885$ & $\overline{0.42}$ & $\overline{0.41}$ & $\overline{-76}$ & 0.10 & 0.16 & & 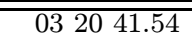 & 052334.6 & $7 \overline{705.6}$ & $\overline{7715.2}$ \\
\hline & 8440 & 0.27 & 0.27 & 47 & 0.06 & 0.07 & & 41.54 & 34.2 & 413.9 & 466.9 \\
\hline & 14940 & 0.18 & 0.14 & 66 & 0.10 & 0.20 & & 41.52 & 34.3 & 157.8 & 201.5 \\
\hline \multirow[t]{6}{*}{$0358+004$} & 4885 & 0.41 & 0.40 & 65 & 0.3 & 0.2 & $\mathrm{a}$ & 035833.36 & $\begin{array}{lll}00 & 28 & 11.2\end{array}$ & 246.6 & 311.8 \\
\hline & & & & & & & $\mathrm{c}$ & 33.31 & 10.8 & 122.2 & 154.0 \\
\hline & & & & & & & d & 33.12 & 08.6 & 3.2 & 5.9 \\
\hline & 8440 & 0.30 & 0.28 & 51 & 0.07 & 0.06 & $\mathrm{a}$ & 33.35 & 11.3 & 144.4 & 209.5 \\
\hline & & & & & & & c & 33.31 & 10.8 & 87.2 & 107.5 \\
\hline & & & & & & & d & 33.11 & 08.7 & 1.0 & 4.5 \\
\hline \multirow[t]{9}{*}{$0809-056$} & 4885 & 0.43 & 0.37 & -0.7 & 0.08 & 0.09 & $\mathrm{a}$ & 080935.53 & $\begin{array}{lll}-05 & 40 & 19.3\end{array}$ & 186.2 & 303.6 \\
\hline & & & & & & & b & 35.45 & 19.4 & 2.3 & 2.3 \\
\hline & & & & & & & $\mathrm{c}$ & 35.19 & 20.2 & 85.4 & 151.2 \\
\hline & 8440 & 0.24 & 0.23 & 37 & 0.02 & 0.03 & $\mathrm{a}$ & 35.51 & 19.6 & 79.8 & 170.8 \\
\hline & & & & & & & $\mathrm{b}$ & 35.42 & 19.8 & 2.4 & 2.7 \\
\hline & & & & & & & $\mathrm{c}$ & 35.17 & 20.5 & 45.3 & 80.2 \\
\hline & 14490 & 0.13 & 0.12 & 42 & 0.06 & 0.13 & $\mathrm{a}$ & 35.52 & 19.6 & 23.6 & 66.6 \\
\hline & & & & & & & b & 35.42 & 19.7 & 1.7 & 1.7 \\
\hline & & & & & & & c & 35.17 & 20.5 & 18.5 & 27.1 \\
\hline \multirow[t]{6}{*}{$1239-044$} & 4885 & 0.43 & 0.38 & -32 & 0.08 & 0.09 & $\mathrm{a}$ & 123945.10 & -042951.2 & 60.2 & 336.8 \\
\hline & & & & & & & b & 44.78 & 54.60 & 291.8 & 634.3 \\
\hline & 8440 & 0.34 & 0.32 & 48 & 0.08 & 0.05 & $\mathrm{a}$ & 45.10 & 51.4 & 29.2 & 180.1 \\
\hline & & & & & & & b & 44.82 & 54.7 & 158.8 & 332.0 \\
\hline & 14490 & 0.12 & 0.12 & 45 & 0.10 & 0.18 & $\mathrm{a}$ & 45.08 & 51.0 & 3.5 & 72.5 \\
\hline & & & & & & & $\mathrm{b}$ & 44.82 & 54.7 & 31.2 & 151.4 \\
\hline \multirow[t]{16}{*}{$1422+202$} & 4885 & 0.46 & 0.36 & -76 & 0.08 & 0.12 & $\mathrm{a}$ & 142237.47 & 201400.5 & 65.2 & 157.1 \\
\hline & & & & & & & b & 37.50 & 1357.6 & 33.5 & 38.2 \\
\hline & & & & & & & c & 37.48 & 54.8 & 14.0 & 31.0 \\
\hline & & & & & & & d & 37.47 & 52.5 & 4.3 & 8.2 \\
\hline & & & & & & & $\mathrm{e}$ & 37.50 & 49.5 & 193.6 & 319.6 \\
\hline & & & & & & & $\mathrm{f}$ & 37.79 & 47.8 & 1.9 & 25.9 \\
\hline & 8440 & 0.30 & 0.27 & 50 & 0.06 & 0.05 & $\mathrm{a}$ & 142237.47 & 201400.5 & 28.6 & 87.1 \\
\hline & & & & & & & $\mathrm{b}$ & 142237.51 & 1357.5 & 26.6 & 27.0 \\
\hline & & & & & & & $\mathrm{c}$ & 37.48 & 57.2 & 7.5 & 21.9 \\
\hline & & & & & & & d & 37.48 & 52.45 & 2.5 & 5.0 \\
\hline & & & & & & & $\mathrm{e}$ & 37.51 & 49.4 & 102.1 & 209.0 \\
\hline & & & & & & & $\mathrm{f}$ & 37.76 & 47.9 & 0.8 & 17.4 \\
\hline & 14940 & 0.17 & 0.15 & 50 & 0.12 & 0.21 & $\mathrm{a}$ & 142237.47 & 201400.4 & 5.8 & 18.5 \\
\hline & & & & & & & b & 37.51 & 1357.5 & 15.0 & 18.7 \\
\hline & & & & & & & $\mathrm{c}$ & 37.49 & 54.7 & 1.6 & 2.1 \\
\hline & & & & & & & $\mathrm{e}$ & 37.51 & 49.4 & 34.0 & 92.2 \\
\hline \multirow[t]{7}{*}{$1741+279$} & 8440 & 0.19 & 0.19 & 44 & 0.06 & 0.05 & $\mathrm{a}$ & 174157.91 & 275410.2 & 5.1 & 43.1 \\
\hline & & & & & & & $\mathrm{b}$ & 57.89 & 04.8 & 73.3 & 81.9 \\
\hline & & & & & & & c & 57.91 & 04.7 & 102.0 & 110.8 \\
\hline & & & & & & & d & 58.01 & 00.9 & 0.6 & 6.3 \\
\hline & 14490 & 0.17 & 0.16 & 47 & 0.10 & 0.18 & $\mathrm{a}$ & 57.91 & 10.3 & 1.9 & 5.7 \\
\hline & & & & & & & $\mathrm{b}$ & 57.89 & 04.8 & 41.0 & 48.5 \\
\hline & & & & & & & $\mathrm{c}$ & 57.91 & 04.7 & 164.9 & 170.7 \\
\hline \multirow[t]{2}{*}{$2033+187$} & 8440 & 0.34 & 0.21 & 75 & 0.04 & 0.05 & & 203318.03 & 184640.1 & 174.0 & 178.8 \\
\hline & 14940 & 0.19 & 0.13 & 70 & 0.10 & 0.22 & & 18.03 & 40.0 & 75.0 & 75.2 \\
\hline \multirow[t]{4}{*}{$2147+145$} & 8440 & 0.35 & 0.22 & 78 & 0.04 & 0.06 & $\mathrm{a}$ & 214759.30 & 143544.7 & 369.1 & 378.8 \\
\hline & & & & & & & $\mathrm{b}$ & 59.29 & 44.9 & 13.7 & 16.1 \\
\hline & 14940 & 0.19 & 0.13 & 74 & 0.15 & 0.22 & a & 59.30 & 44.7 & 154.6 & 165.1 \\
\hline & & & & & & & $\mathrm{b}$ & 59.28 & 45.0 & 4.3 & 4.6 \\
\hline
\end{tabular}

the observing frequency in MHz; Columns 3 to 5: major axis, minor axis (both in arcsec) and the PA in degrees of the restoring beam major axis; Column 6: the rms noise in the total intensity map far from the source of emission; Column $7:-$ the rms noise $\sqrt{\sigma_{Q}^{2}+\sigma_{U}^{2}}$, where $\sigma_{Q}$ and $\sigma_{U}$ are the rms noise on the blank sky in the distributions of the Stokes parameters $Q$ and $U$; Column 8 component label; Columns 9 and 10: RA and Dec. of the component peak; Column 11: peak flux density (mJy) of the component; Column 12: total flux density (mJy) of the component. The total intensity images at $5 \mathrm{GHz}$ for the sources listed in Table 1 except for $1741+279$, 2033+187 and $2147+145$ were presented in Mantovani et al. (1992). However, observational parameters and derived properties obtained from the VLA observations at $5 \mathrm{GHz}$ are incorporated in Table 1 to aid the reader. 
Table 2. Polarization parameters

\begin{tabular}{|c|c|c|c|c|c|c|c|c|c|c|c|c|c|}
\hline \multirow[t]{2}{*}{$\overline{\text { Source }}$} & \multirow[t]{2}{*}{ O.I. } & \multirow[t]{2}{*}{$z$} & \multirow[t]{2}{*}{$\mathrm{C}$} & \multicolumn{3}{|c|}{$\overline{\mathrm{PA}}$} & \multirow[t]{2}{*}{$R M$} & \multirow{2}{*}{$\begin{array}{r}R M \times \\
(1+z)^{2} \\
\end{array}$} & \multicolumn{3}{|c|}{$\% \mathrm{Pol}$} & \multirow{2}{*}{$\begin{array}{r}\mathrm{DP} \\
6-3.6 \\
\end{array}$} & \multirow{2}{*}{$\begin{array}{r}\mathrm{DP} \\
3.6-2 \\
\end{array}$} \\
\hline & & & & $6 \mathrm{~cm}$ & $3.6 \mathrm{~cm}$ & $2 \mathrm{~cm}$ & & & $6 \mathrm{~cm}$ & $3.6 \mathrm{~cm}$ & $2 \mathrm{~cm}$ & & \\
\hline$\overline{0358+004}$ & $\bar{G}$ & 0.426 & $\mathrm{a}$ & $\overline{117 \pm 2}$ & $\overline{115 \pm 2}$ & & $\overline{0}$ & 0 & 74.9 & 9.5 & & $\overline{c 0.52}$ & \\
\hline \multirow{2}{*}{$0809-056$} & & & $\mathrm{a}$ & $70 \pm 1$ & $72 \pm 1$ & $71 \pm 1$ & -7 & & 9.5 & 10.9 & 11.3 & 0.87 & 0.96 \\
\hline & & & c & $87 \pm 1$ & $90 \pm 1$ & $92 \pm 1$ & -25 & & 7.5 & 11.0 & 13.1 & 0.68 & 0.84 \\
\hline \multirow[t]{2}{*}{$1239-044$} & G & 0.480 & $\mathrm{a}$ & $86 \pm 1$ & $88 \pm 1$ & $90 \pm 1$ & -19 & -42 & 13.5 & 14.7 & ${ }^{a} 11.6$ & 0.92 & 1.3 \\
\hline & & & b & $2 \pm 1$ & $1 \pm 2$ & $-8 \pm 2$ & 24 & 52 & 2.3 & 3.7 & 5.3 & 0.62 & 0.70 \\
\hline \multirow[t]{4}{*}{$1422+202$} & Q & 0.871 & $\mathrm{a}$ & $26 \pm 6$ & $33 \pm 3$ & $42 \pm 4$ & -75 & -263 & 6.3 & 6.8 & 7.5 & 0.93 & 0.91 \\
\hline & & & $\mathrm{b}$ & $104+1$ & $\begin{array}{l}112 \pm 1 \\
108+1\end{array}$ & & -28 & -98 & 34.2 & $\begin{array}{r}0.8 \\
34.2\end{array}$ & & 1 & \\
\hline & & & d & $\begin{array}{r}96 \pm 1 \\
96 \pm 1\end{array}$ & $\begin{array}{l}100 \pm 1 \\
102 \pm 1\end{array}$ & & $\begin{array}{l}-20 \\
-42\end{array}$ & -147 & $\begin{array}{l}3.2 \\
39.0\end{array}$ & $\begin{array}{l}54.2 \\
63.3\end{array}$ & & $\begin{array}{r}1 \\
0.62\end{array}$ & \\
\hline & & & e & $150 \pm 3$ & $160 \pm 3$ & $156 \pm 1$ & -39 & -137 & 10.2 & 10.9 & $a_{8.2}$ & 0.94 & 1.3 \\
\hline \multirow[t]{3}{*}{$1741+279$} & $\mathrm{Q}$ & 0.372 & a & & $51 \pm 6$ & & & & & 18.3 & & & \\
\hline & & & $\mathrm{b}$ & & $75 \pm 6$ & $74 \pm 6$ & 0 & 0 & & 7.6 & 9.6 & & 0.79 \\
\hline & & & $\mathrm{c}$ & & $42 \pm 5$ & $35 \pm 5$ & 135 & 254 & & 4.0 & $a_{1.8}$ & & 2.2 \\
\hline
\end{tabular}

$a$ : see notes on individual sources.

\subsection{VLA polarimetry}

The images obtained at $8.4 \mathrm{GHz}$ and $15 \mathrm{GHz}$ were convolved with a two-dimensional, circularly-symmetric Gaussian with a width of $\sim 0.4$ arcsec. This approximates the resolution of the synthesized beam of the VLA Aarray at $5 \mathrm{GHz}$. Parameters were calculated from the new images. Table 2 contains the following information: Column 1: source name; Column 2: optical identification; Column 3: the measured redshift; Column 4: component label; Columns 5 to 7: PA in degrees of the electric field vector at the peak of polarized emission $( \pm 1 \mathrm{rms}$ error calculated from the distribution of PAs in a small box around the peak of polarized emission) at 5, 8.4 and 15 GHz respectively; Column 8: the Rotation Measure defined as $R M=\mathrm{d} \phi(\lambda) / \mathrm{d}\left(\lambda^{2}\right)$ in $\mathrm{rad} \mathrm{m}^{-2}$, where $\phi(\lambda)$ is the PA at wavelength $\lambda$. The $R M$ is estimated by fitting the points with a linear least-squares fit; when computed between two frequencies $R M$ suffers from an $n \pi$ ambiguity. Column 9: the $R M$ corrected for redshift; Columns 10 to 12: polarization percentage at $5,8.4$ and $15 \mathrm{GHz}$ respectively; Columns 13 and 14: the depolarization index, defined as the ratio of the fractional polarization at the longer wavelength to the fractional polarization at the shorter wavelength.

\subsection{Notes on individual sources}

\subsection{1. $0320+053$}

This source appears, at best, to be only slightly resolved even at $15 \mathrm{GHz}$. At a redshift of 0.575 (Heckman et al. 1994), the linear diameter is $<0.3 \mathrm{kpc}\left(H_{0}=\right.$ $\left.100 \mathrm{~km} \mathrm{~s}^{-1} \mathrm{Mpc}^{-1} ; q_{0}=1\right)$. There was no detection of polarized emission above the noise levels at any of the observing frequencies. The spectral index $\left(S \propto \nu^{-\alpha}\right)$ ranges from $\alpha=0.89$ between 5 and $8.4 \mathrm{GHz}$ to $\alpha=1.46$ between 8.4 and $15 \mathrm{GHz}$.

\subsection{2. $0358+004$ (3C 99)}

The image obtained for $3 \mathrm{C} 99$ at $8.4 \mathrm{GHz}$ is presented in Fig. 1 and shows the well-known asymmetric triple structure (Mantovani et al. 1990). The $8.4 \mathrm{GHz}$ image shows that the polarized emission comes mainly from component A. The jet region (i.e. the region between components $\mathrm{A}$ and $\mathrm{C}$ ) is also slightly polarized with the magnetic field aligned with the jet axis. The $15 \mathrm{GHz}$ data were affected by interference and are not presented here. In the earlier investigation of its structure by Mantovani et al. (1990), the A component was shown to contain a hot spot with a compact bright feature which has been detected in VLBI observations. Additionally, it was suggested that component $\mathrm{C}$ contains the nucleus.

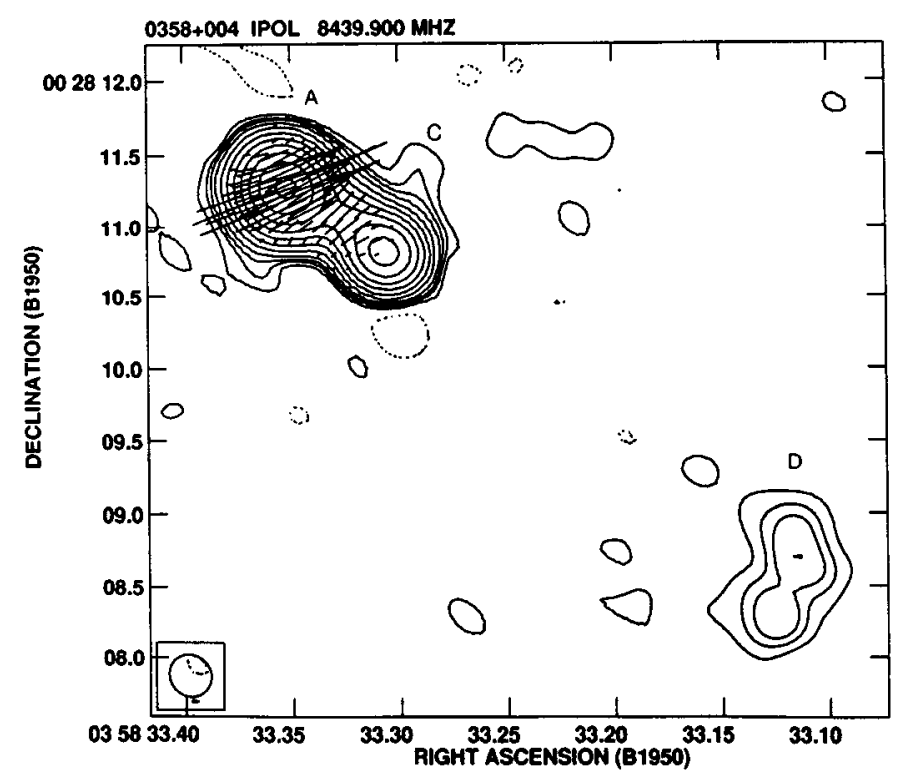

Fig. 1. VLA image of $0358+004$ at $8.4 \mathrm{GHz}$. contours are at $-0.2,0.2,0.4,0.6,1,2,4,8,16,32,64,128,256$ mJy beam $^{-1}$. The peak flux density is $144.4 \mathrm{mJy}^{\text {beam }}{ }^{-1}$. A vector length of $1^{\prime \prime}=10$ mJy beam ${ }^{-1}$ 


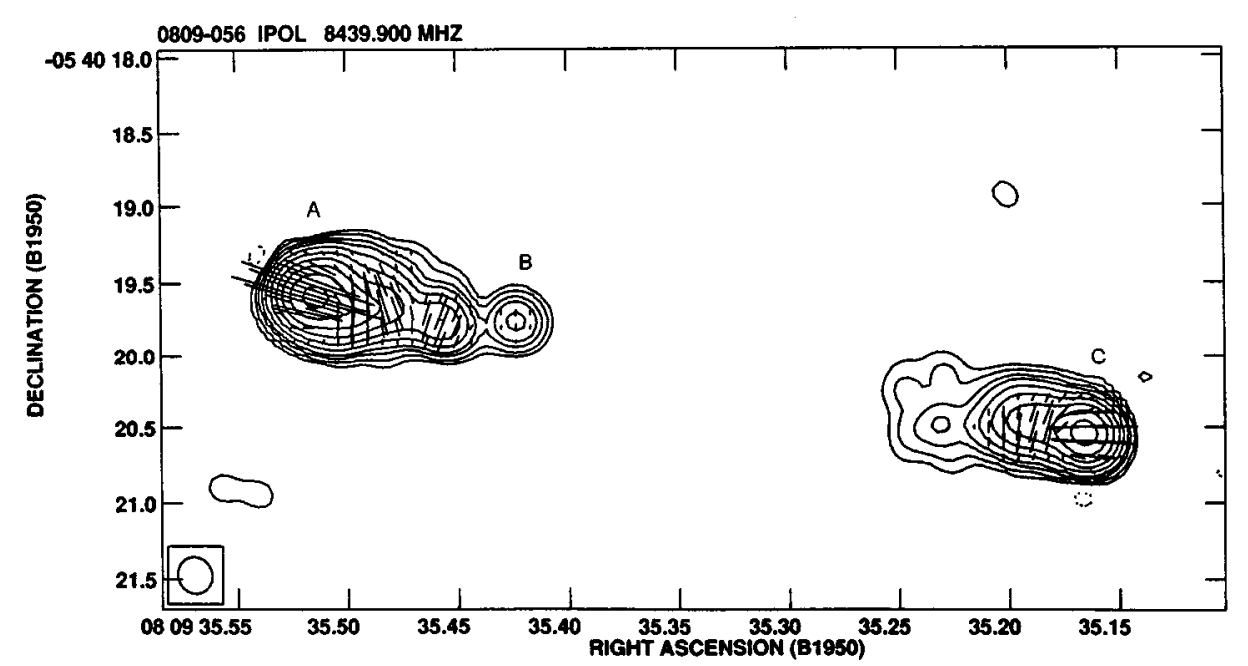

Fig. 2. VLA image of $0809-056$ at $8.4 \mathrm{GHz}$. contours are at $-0.15,0.15,0.3,0.6,1,2,4,8,16,32,64,128 \mathrm{mJy}^{\mathrm{beam}}{ }^{-1}$. The peak flux density is $79.8 \mathrm{mJy}_{\text {beam }}^{-1}$. A vector length of $1^{\prime \prime}=10 \mathrm{mJy}_{\text {beam }}^{-1}$

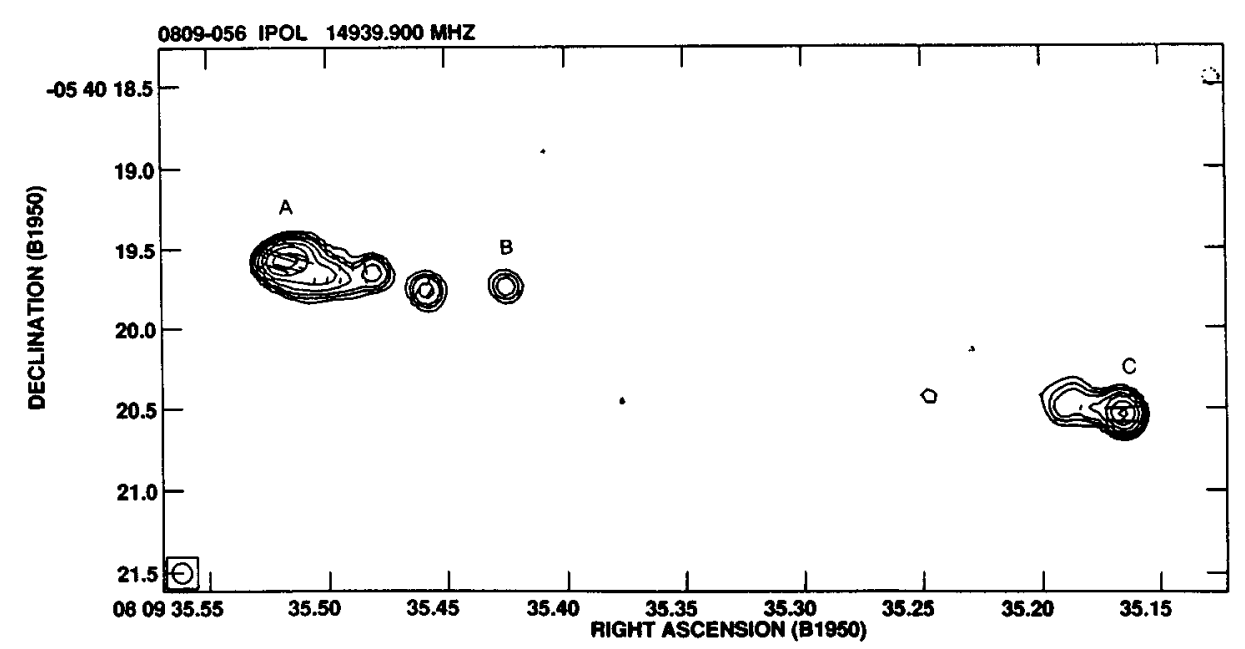

Fig. 3. VLA image of $0809-056$ at $15 \mathrm{GHz}$. contours are at $-0.3,0.3,0.6,1,2,4,8,16,32$ mJy beam ${ }^{-1}$. The peak flux density is $23.6 \mathrm{mJy}$ beam $^{-1}$. A vector length of $1^{\prime \prime}=12.5 \mathrm{mJy}_{\text {beam }}{ }^{-1}$

\subsection{3. $0809-056$}

The two images obtained for $0809-056$ at $8.4 \mathrm{GHz}$ and $15 \mathrm{GHz}$ (Figs. 2 and 3 respectively) confirm the double structure found at $5 \mathrm{GHz}$. The point-like component labelled B is likely to be the core of the source since it has a flat spectrum. It is also not polarized at the detection threshold of the present observations with an upper limit of about 3.5 per cent at $8.4 \mathrm{GHz}$. If this is the case, the source is asymmetric, with the further component $(\mathrm{C})$ more depolarized, while going from shorter to longer wavelengths, and with a larger Faraday rotation than $\mathrm{A}$. The more extended features in the lobes are not seen at $\lambda 2 \mathrm{~cm}$. The extended emission from the lobe, which is polarized at both $\lambda 6$ and $\lambda 4 \mathrm{~cm}$, is below the detection limits at $\lambda 2 \mathrm{~cm}$ even in the image smoothed to the $\lambda 6 \mathrm{~cm}$ resolution.
The magnetic field is aligned along the two components and suddenly changes direction by about $90^{\circ}$ in the hot spots. The spectral indices of the two lobes are as steep as $1.3-1.4$.

\subsection{4. $1239-044$}

This source shows a double lobed-structure that is asymmetric with a flux density ratio between the two lobes of about two, with lobe B being the brighter one. In Figs. 4 and 5 we present the 8.4 and $15 \mathrm{GHz}$ images respectively. There is no indication of a component which could be the candidate for the nucleus. In the components, the $\boldsymbol{E}$ vectors suggest a circumferential field in the outer edges of the lobes but there are significant changes in the PAs of the vectors within the lobes. The Faraday rotation is 
generally low, with values $<100 \mathrm{rad} \mathrm{m}^{-2}$, over the components. There is a suggestion of a large $R M$ in the region around RA 123944.85 and Dec -04 2954.3 in lobe B with a value of about $440 \mathrm{rad} \mathrm{m}^{-2}$ in the source rest frame. However, this is close to one of the regions where the PAs change significantly across the lobe, and the high $R M$ needs to be confirmed from more sensitive observations of higher resolution. The lobe $\mathrm{A}$ is heavily resolved at $15 \mathrm{GHz}$. The diffuse innermost part of the lobe is below the detection limit even in the smoothed image.

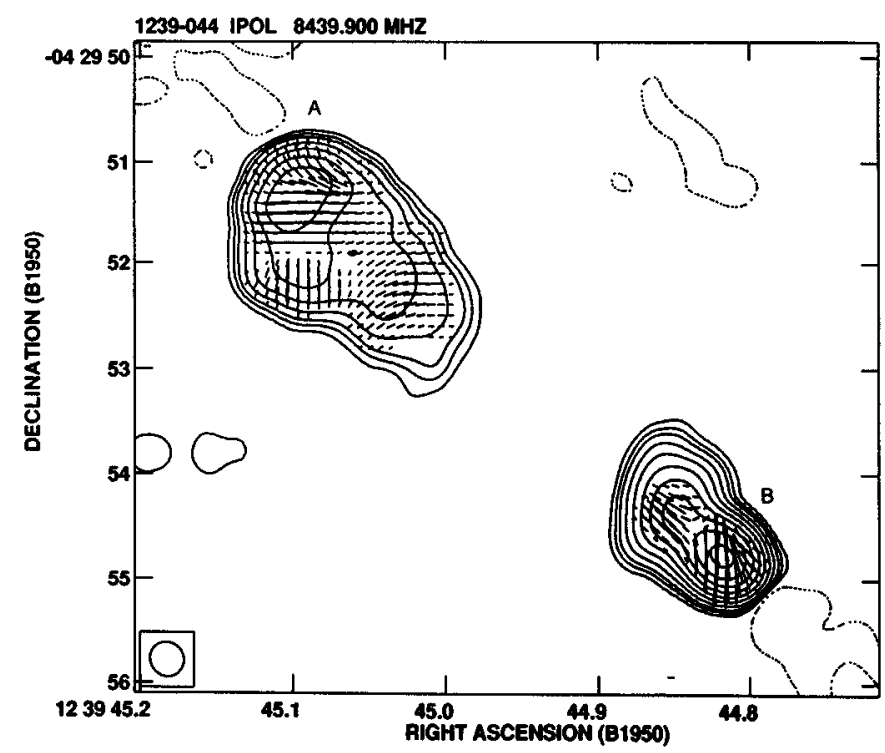

Fig. 4. VLA image of $1239-044$ at $8.4 \mathrm{GHz}$. contours are at $-0.3,0.3,0.6,1,2,4,8,16,32,64,128,256$ mJy beam $^{-1}$. The peak flux density is 158.8 mJy beam $^{-1}$. A vector length of $1^{\prime \prime}=$ 10 mJy beam ${ }^{-1}$

\subsection{5. $1422+202$}

This asymmetric source shows a long collimated one-sided jet. The core (component $\mathrm{B}$ ) has a very low value of percentage polarization at $8 \mathrm{GHz}$ (Fig. 6) which is likely to originate from the emerging part of the jet (see the $15 \mathrm{GHz}$ image; Fig. 7). Polarized emission is detected all along the jet with the polarization percentage being as high as $30-60 \%$ in components $\mathrm{C}$ and $\mathrm{D}$. The magnetic field looks quite ordered parallel to the jet axis and it changes direction in the hot spot (component E). The polarized emission at $15 \mathrm{GHz}$ from component $\mathrm{E}$ is lower than those found at lower frequencies. The reason is possibly that the emission from that component, mainly along the ridge, is resolved out or below the sensitivity of these observations. The $R M$ along the jet is never larger than $130 \mathrm{rad} \mathrm{m}^{-2}$ in the source rest frame. The lobe A on the counter-jet side is less polarized. Its emission is more diffuse and is located closer to the nucleus.

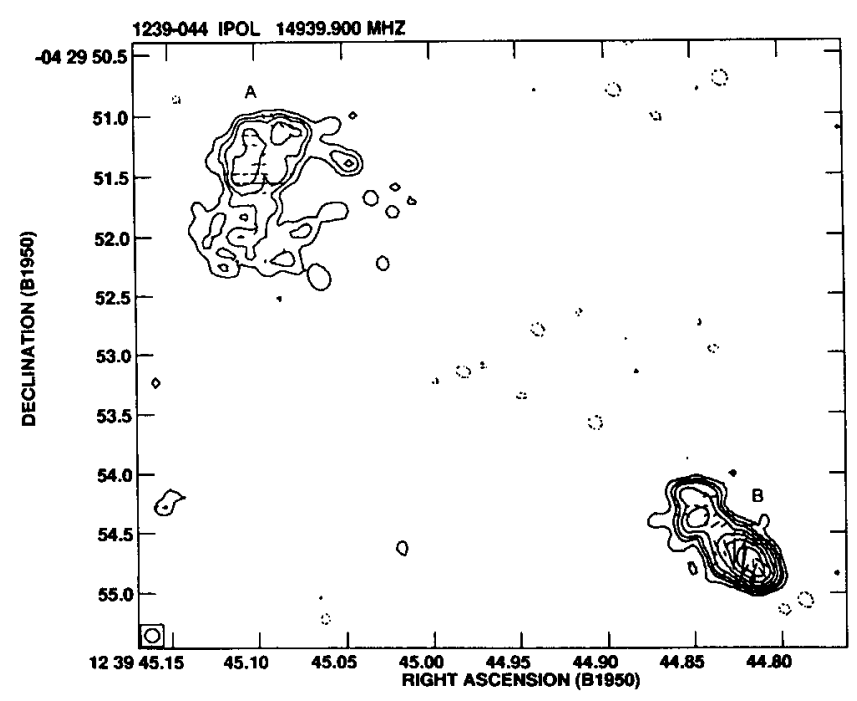

Fig. 5. VLA image of $1239-044$ at $15 \mathrm{GHz}$. contours are at $-0.3,0.3,0.6,1,2,4,8,16,32 \mathrm{mJy}_{\text {beam }}{ }^{-1}$. The peak flux density is $31.2 \mathrm{mJy}$ beam $^{-1}$. A vector length of $1^{\prime \prime}=12.5 \mathrm{mJy}$ beam $^{-1}$

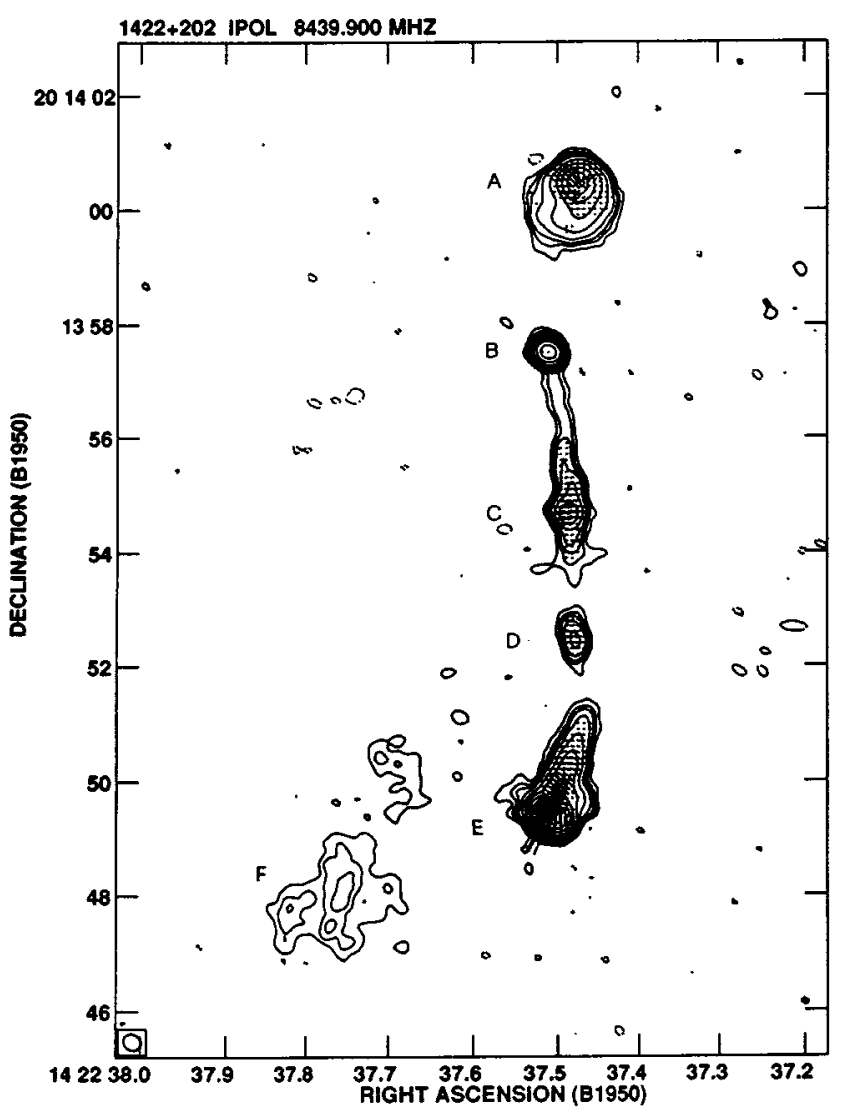

Fig. 6. VLA image of $1422+202$ at $8.4 \mathrm{GHz}$. contours are at $-0.2,0.2,0.4,0.6,1,2,4,8,16,32,64,128$ mJy beam $^{-1}$. The peak flux density is $102.1 \mathrm{mJy}^{\text {beam }}{ }^{-1}$. A vector length of $1^{\prime \prime}=$ 10 mJy beam $^{-1}$ 


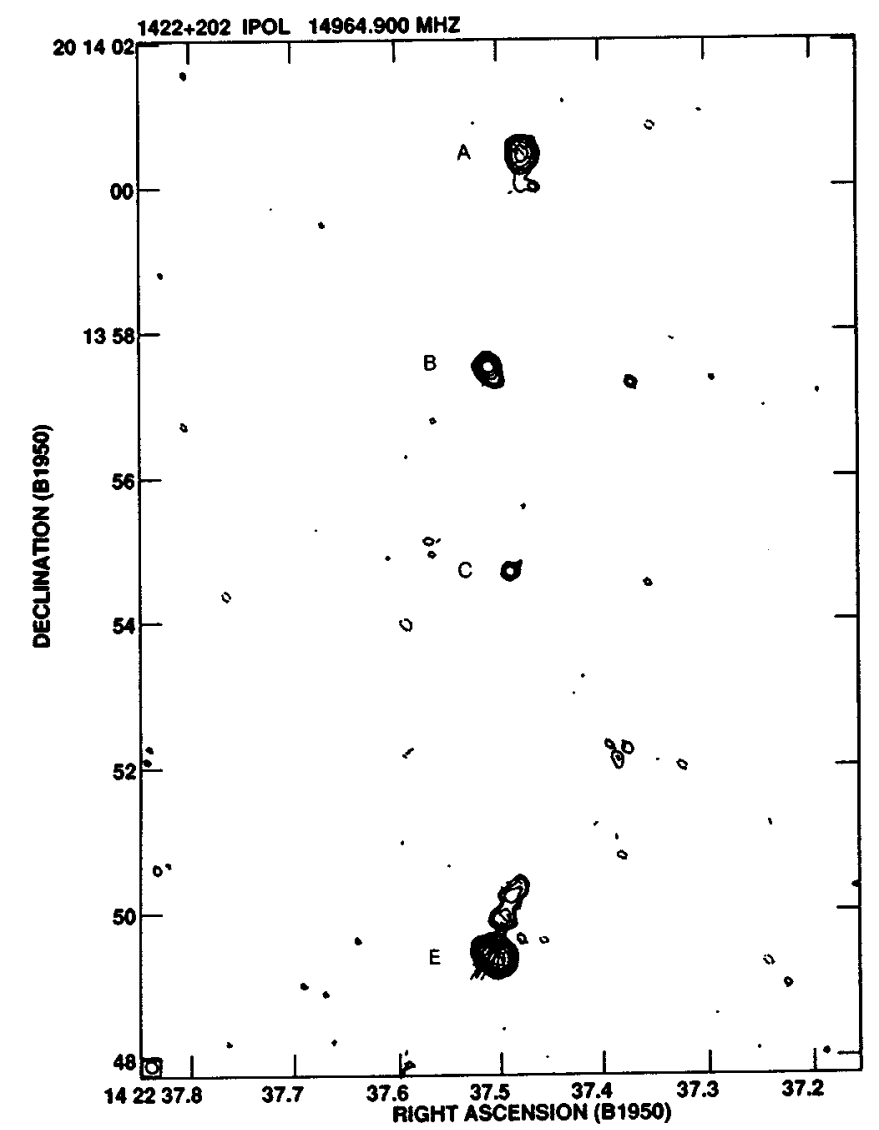

Fig. 7. VLA image of $1422+202$ at $15 \mathrm{GHz}$. contours are at $-0.3,0.3,0.6,1,2,4,8,16,32 \mathrm{mJy}^{\text {beam }}{ }^{-1}$. The peak flux density is $34.0 \mathrm{mJy}^{\text {beam }}{ }^{-1}$. A vector length of $1^{\prime \prime}=10 \mathrm{mJy}$ beam $^{-1}$

\subsection{6. $1741+279$}

The image of this source at $8.4 \mathrm{GHz}$ (Fig. 8) confirms the triple structure seen at $408 \mathrm{MHz}$ in the MERLIN map by Mantovani et al. (1992). At $15 \mathrm{GHz}$ (Fig. 9) we detect only the central components and the hot spot at the end of the northern jet. The central component is resolved in the VLA observations at higher resolution. The $\mathrm{C}$ component has an inverted spectral index and is likely to host the nucleus of the source although it has polarized emission. The component $\mathrm{B}$ exhibits marginal depolarization between $15 \mathrm{GHz}$ to $8.4 \mathrm{GHz}$ while component $\mathrm{C}$ is more stongly polarized at the lower frequency. However, the values reported in Table 2 have to be taken with care since the two components are barely separated. Since the $R M$ s of components $\mathrm{B}$ and $\mathrm{C}$ are consistent with small values, within the errors, the inferred magnetic field is initially along the line connecting the peaks of emission of the two components, and later changes direction gradually to point towards the northern component A. There appears to be a large bend in the jet close to the nucleus. The jet later points northwards and has a wiggling collimated structure.

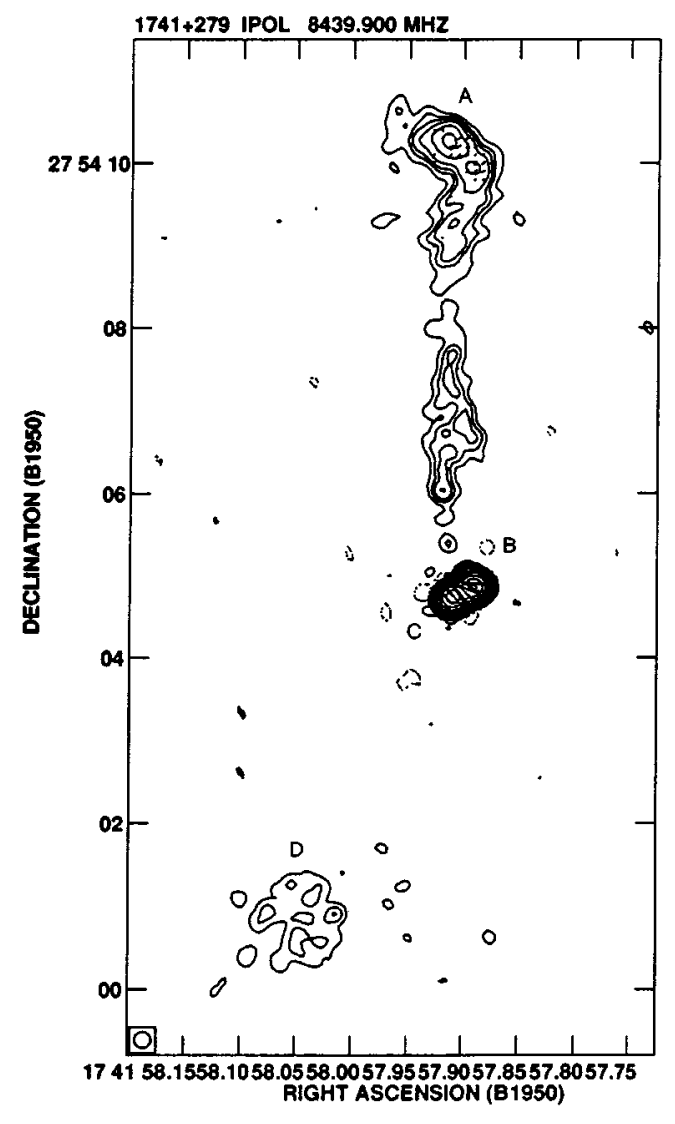

Fig. 8. VLA image of $1741+279$ at $8.4 \mathrm{GHz}$. contours are at $-0.2,0.2,0.4,0.6,1,2,4,8,16,32,64,128$ mJy beam $^{-1}$. The peak flux density is $100.4 \mathrm{mJy}_{\text {beam }}{ }^{-1}$. A vector length of $1^{\prime \prime}=$ $10 \mathrm{mJy}_{\text {beam }^{-1}}$

\subsection{7. $2033+187$}

The VLA observations confirm that $2033+187$ is unresolved at the sub-arcsecond resolution scale. The emission is not polarized at the sensitivity level of these observations.

\subsection{8. $2147+145$}

This source was found to be unresolved in previous VLA A-array observations at $5 \mathrm{GHz}$ and elongated along PA $159^{\circ}$ at $15 \mathrm{GHz}$ (Cotton 1983). We detect a weak unresolved component north-west of the main component along a PA of $140^{\circ}$ and separated from it by about 0.4 arcsec (Figs. 10 and 11). This new component has a steep spectral index $(\alpha \sim 1.8)$ and lies off-axis by about $90^{\circ}$ with respect to the axis of the VLBI jet (Cotton et al. 1984). No polarized emission was detected in either components.

\section{Discussion}

The data presented in this paper complete the information about a sample of small-sized sources 


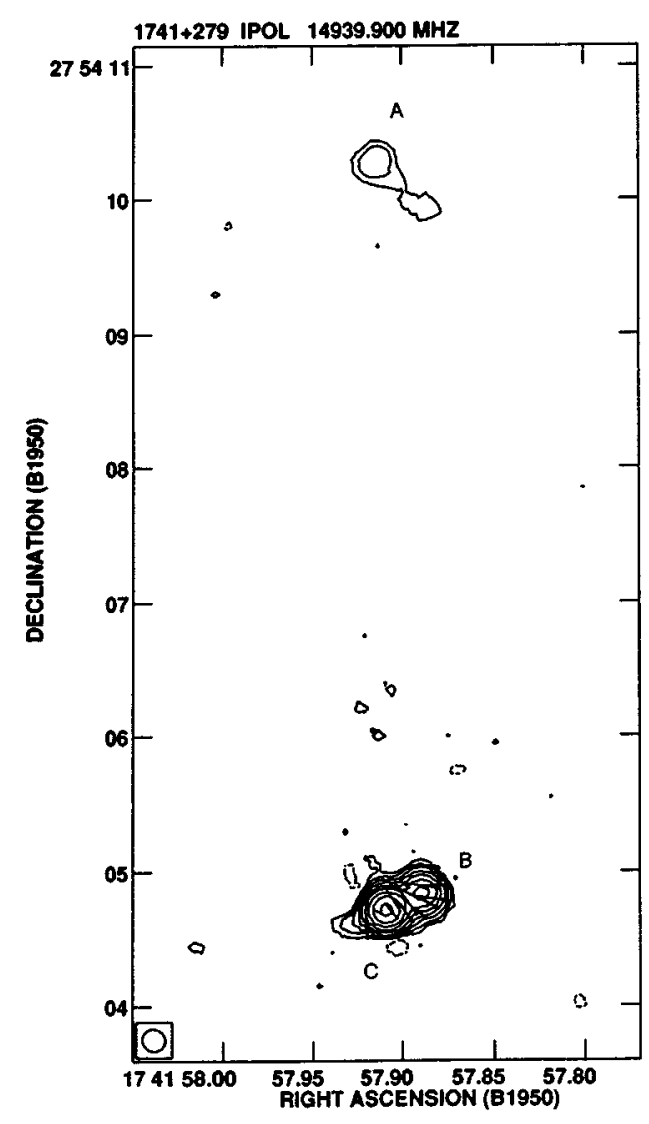

Fig. 9. VLA image of $1741+279$ at $15 \mathrm{GHz}$. contours are at $-0.4,0.4,0.6,1,2,4,8,16,32,64,128 \mathrm{mJy}_{\text {beam }^{-1}}$. The peak flux density is $159.4 \mathrm{mJy}^{\text {beam }}{ }^{-1}$. A vector length of $1^{\prime \prime}=10 \mathrm{mJy}_{\text {beam }^{-1}}$

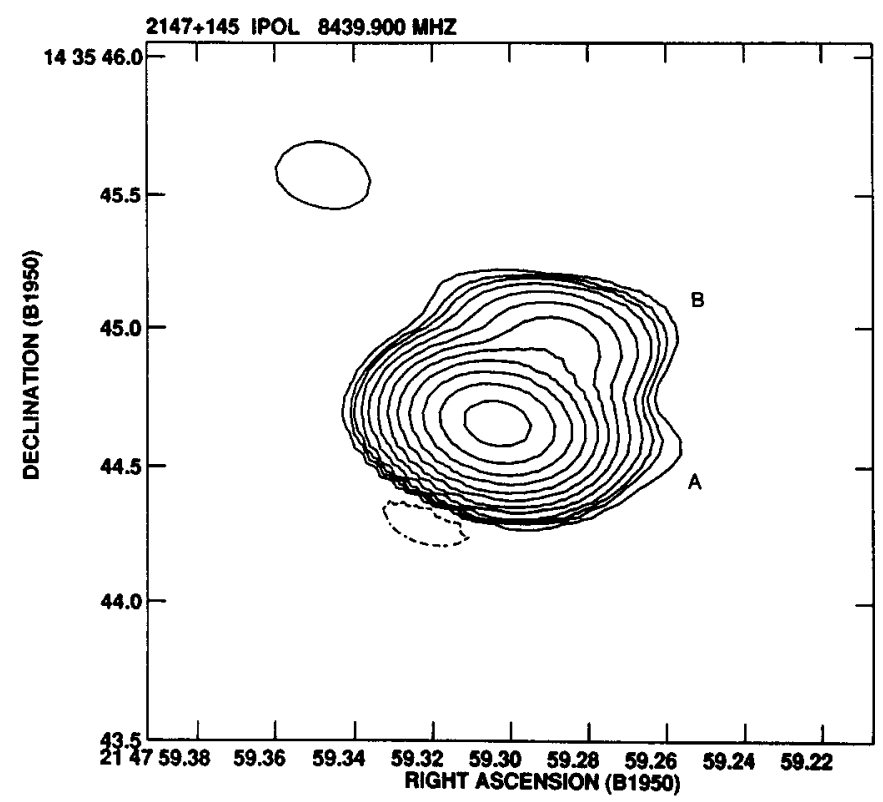

Fig. 10. VLA image of $2147+145$ at $8.4 \mathrm{GHz}$. contours are at $-0.2,0.2,0.4,0.6,1,2,4,8,16,32,64,128,256,512 \mathrm{mJy}$ beam $^{-1}$. The peak flux density is 369.1 mJy beam $^{-1}$

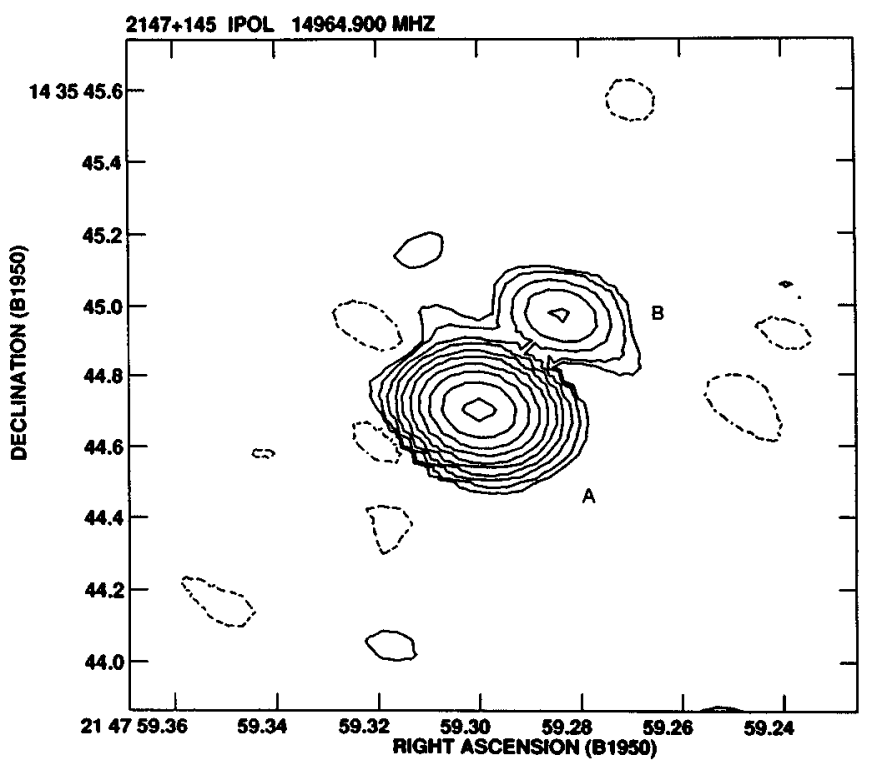

Fig. 11. VLA image of $2147+145$ at $15 \mathrm{GHz}$. contours are at $-0.2,0.2,0.4,1,2,4,8,16,32,64,128,256$ mJy beam $^{-1}$. The peak flux density is $154.6 \mathrm{mJy}^{\text {beam }}{ }^{-1}$

(Mantovani et al. 1992, 1994). The previous paper (Mantovani et al. 1994) presented the results on sources which were generally of small size and had high rotation measures. All but one of the sources discussed in that paper have a linear size $<14 \mathrm{kpc}$. The source $0725+147$ is larger with a projected linear size of $25 \mathrm{kpc}$, and was found to have rotation measures of about +545 and $-2576 \mathrm{rad}$ $\mathrm{m}^{-2}$ for the two lobes in the rest frame of the source. This object is associated with a quasar at a redshift of 1.387 and has an absorption-line system at a similar redshift (Hewitt \& Burbidge 1993).

Among the sources presented here, 0320+053, $2033+187$ and $2147+145$ are the most compact with the former two being unresolved with the VLA A-array at $15 \mathrm{GHz}$. This implies an angular dimension $<100$ mas, which corresponds to a linear size $<0.3 \mathrm{kpc}$ for $z=0.5$. All of these are not polarized at the detection levels of these observations.

Two sources, 0809-056 and 1239-044, have an overall structure that is similar to $0725+147$. However, unlike $0725+147$, they do not show evidence of large rotation measure. The source $0358+004$ also has a triple structure, which is much more asymmetric compared to the above group of objects. All of them have a linear size in the range $20-25 \mathrm{kpc}$, a bit larger than the limit set by Fanti et al. (1995) for a steep spectrum source to be considered a member of the class of Compact Steep-spectrum Sources.

The two last sources in the sample, $1422+202$ and $1741+279$, each show a one-sided jet and an overall asymmetric structure, with a weak lobe of emission on the counter-jet side which is located closer to the nucleus. The 
jets are knotty and the one in $1741+279$ has a wiggling structure which deserves further investigation. Moreover, in the case of $1741+279$ there is a clear indication of a large bend in the jet direction close to the component which is likely to host the nucleus.

The above five sources, including $0725+147$, have a linear size which is somewhat larger (between about 21 and $43 \mathrm{kpc}$ ) than the "required" size of $\leq 15 \mathrm{kpc}$ to be classified as CSSs. They can be interpreted as medium sized objects which are the more evolved versions of the CSSs, which are assumed to be young rather than frustrated objects (Fanti et al. 1995; Readhead et al. 1996).

We have summarized in Table 3 the overall polarization parameters derived for the five polarized sources of the group. It is worth mentioning that the percentage polarization of these sources is rather high (in the range of about $6-13 \%$ at $8.4 \mathrm{GHz}$ ) and that they depolarize going from higher to lower frequencies. The percentage polarization is significantly higher than that found for the compact sources with high RM presented in Mantovani et al. (1994). The four compact sources show a percentage polarization $<3$, while only $0725+147$ has a percentage polarization comparable to the above values. The fact that the percentage polarization shown by some of the components is sometimes lower at $15 \mathrm{GHz}$ than at $8.4 \mathrm{GHz}$ is probably due to the fact that the sensitivity of the observations to the low brightness emission was insufficient in those cases.

Table 3. Polarization parameters of the sources as a whole

\begin{tabular}{lrrrrr}
\hline Source & \multicolumn{1}{c}{$\begin{array}{r}\text { DP } \\
\text { DPol }\end{array}$} \\
\hline \hline $0358+004$ & 3.2 & 6.4 & & 0.5 & \\
$0809-056$ & 8.8 & 13.2 & 18.2 & 0.7 & 0.7 \\
$1239-044$ & 6.2 & 8.3 & 8.1 & 0.7 & 1.0 \\
$1422+202$ & 9.7 & 12.0 & 7.9 & 0.8 & 1.5 \\
$1741+279$ & & 8.5 & 3.4 & & 2.5 \\
\hline
\end{tabular}

Acknowledgements. The authors wish to think the referee, Dr. Ian W.A. Browne for his suggestions. FM thanks Miller Goss, Assistant Director, NRAO, Socorro, for his hospitality during period when part of the work was done. The National Radio Astronomy Observatory is operated by Associated Universities Inc., under cooperative agreement with the National Science Foundation; AIPS is NRAO's Astronomical Image Processing System.

\section{References}

Cotton W.D., 1983, ApJ 271, 51

Cotton W.D., Owen F.N., Geldzahler B.J., et al., 1984, ApJ 277, L41

Fanti R., Fanti C., Schilizzi R.T., et al., 1990, A\&A 231, 333

Fanti C., Fanti R., Dallacasa D., et al., 1995, A\&A 302, 317

Heckman T.M., O'Dea C.P., Baum S.A., Laurikainen E., 1994, ApJ 428, 65

Hewitt A., Burbidge G., 1993, ApJS 87, 451

Inoue M., Tabara H., Kato T., Aizu K., 1995, PASJ 47, 725

Junor W., Mantovani F., Peck A., et al., 1996, in: IAU Symposium No. 175, Ekers R., Fanti C., Padrielli L. (eds.)

Kato T., Tabara H., Inoue M., Aizu K., 1987, Nat 329, 223

Mantovani F., Saikia D.J., Browne I.W.A., et al., 1990, MNRAS 245, 427

Mantovani F., Junor W., Fanti R., et al., 1992, MNRAS 257, 353

Mantovani F., Junor W., Fanti R., et al., 1994, A\&A 292, 59

Pearson T.J., Perley R.A., Readhead A.C.S., 1985, AJ 90, 738

Readhead A.C.S., Taylor G.B., Pearson T.J., Wilkinson P.N., 1996, AJ 460, 634

Saikia D.J., Singal A.K., Cornwell T.J., 1987, MNRAS 224, 379

Saikia D.J., Jeyakumar S., Wiita P.J., et al., 1995, MNRAS 276,1215

Taylor G.B., Inoue M., Tabara H., 1992, A\&A 264, 421

Thompson A.R., Clark B.G., Wade C.M., Napier P.J., 1980, ApJS 44, 151 